BIS-RS-2015-45

\title{
AN ANALYTICAL, LOW-COST DEPLOYMENT STRATEGY FOR SATELLITE CONSTELLATIONS
}

\author{
Ciara McGrath, Emma Kerr and Malcolm Macdonald \\ University of Strathclyde, United Kingdom
}

This work proposes a novel method for the deployment of a constellation of nano-satellites into Low Earth Orbit by using carrier vehicles to deliver the nano-satellites into the required orbit positions. The analytical solution presented allows for rapid exploration of the design space and a direct optimisation of the deployment strategy to minimise the time for complete constellation deployment. Traditionally, the deployment of satellite constellations requires numerous launches - at least one per orbital plane - which can be costly. Launching as a secondary payload may offer significant cost reductions, but this comes at the price of decreased control over the launch schedule and final orbit parameters. The analytical method presented here allows for the optimal positioning of the orbit planes of the constellation to be determined and the minimum time for deployment determined as a function of the manoeuvre $\Delta \mathrm{V}$. The effect of atmospheric drag on the manoeuvre propellant cost is also considered to ensure a realistic deployment scenario. A case study considering three constellation designs is presented which compares the cost of deployment using traditional launch methods with that of deploying the constellation using carrier vehicles. The results of this study show a significant reduction in cost when using the carrier vehicles on a dedicated launch, compared with launching the satellites individually. Most significantly, the launch cost when using carrier vehicles is primarily determined by the total number of satellites in the constellation, rather than the number of orbital planes. Thus, the carrier vehicle deployment strategy would allow for constellations with a large number of planes to be deployed for a fraction of the equivalent cost if traditional launch methods were used.

\section{INTRODUCTION}

Nano-satellites in general are becoming increasingly common with almost 150 nanosatellites currently operational and more than 400 launched in total since 1998 [1]. Nano-satellites are satellites with a mass of $1-10 \mathrm{~kg}$ and include satellites conforming to the popular CubeSat standard [2]. The increasing flight heritage associated with their increased use means that they are no longer confined to Universities and educational institutions. Larger space organisations such as NASA, Boeing and The Aerospace Corporation are also building and launching their own nano-satellites either for technology demonstration or scientific research [3]. With the rapidly increasing capabilities of nano-satellites, their performance has now reached a point where they are capable of supporting Earth Observation (EO) missions. In particular, a large constellation of nano-satellites could prove valuable in supporting existing Earth Observation systems by reducing the burden on current EO satellites and providing data with a high temporal resolution that cannot be achieved by existing systems [4-7].

In line with these developments, the Advanced Space Concepts Laboratory at the University of Strathclyde has carried out a preliminary mission design study considering a constellation of nanosatellites capable of rapidly performing measurements of tropospheric properties to support real-time 'nowcasting' of severe weather [8]. The constellation proposed would be required to provide high temporal resolution and low data latency, while still remaining low cost. To fulfil these mission requirements the study proposed the use of CubeSats deployed in a constellation and performs a multi-attribute utility-cost trade-off analysis to identify the best value for money constellation architecture. One of the key costs identified is the launch cost which, in the case of a dedicated launch, increases as the number of satellite planes increases, and as the number of satellites per plane decreases. Rideshare launches are also considered, in which the satellites would be launched as secondary payloads alongside a primary customer, but the lack of control over the final orbit makes the achievable constellation performance unpredictable and reliance on their services undesirable.

As demonstrated by the FORMOSAT3/COSMIC mission in 2006, an alternative method of constellation deployment is to launch a number of satellites into a single orbit plane and then separate the orbital planes of the satellites to achieve the required separation of the RightAscension of the Ascending Node (RAAN) and argument of latitude $[9,10]$. The propellant mass associated with such a deployment manoeuvre can be reduced by making use of low-thrust propulsion and utilising the natural perturbations of the Earth's $\mathrm{J}_{2}$ effect to produce the desired RAAN change, at the cost of a longer manoeuvre time [11].

This method of deployment has the potential to reduce the number of launches required to populate 
a constellation and thus reduce overall mission cost. Traditionally, the design of constellation reconfiguration manoeuvres has been handled using numerical methods, often requiring the use of complex optimisation techniques $[12,13]$. A semianalytical method has also been proposed, but it requires full knowledge of the satellite orbit parameters before and after reconfiguration, meaning it is not ideal for performing a trade-space exploration [14].

A fully analytical solution describing satellite manoeuvres which could be used to reconfigure a constellation through Right Ascension of the Ascending Node (RAAN) and Argument of Latitude (AoL) has previously been presented by the authors [15]. This method is extended here to optimise the satellite deployment manoeuvres for a number of constellations designs and ultimately provide a comparison of the designs in terms of deployment time and overall cost.

\section{GENERAL METHOD}

Analysis of the deployment of a constellation of nano-satellites is done using the fully analytical method previously described by the authors [15]. In this method, two manoeuvres are considered independently; one manoeuvre to change the RAAN of a satellite, and one to change the AoL. Both manoeuvres are performed by varying the altitude of the satellites relative to each other, creating a variation in the rate of change of RAAN and AoL between the satellites. The most general case of this is considered here in which the satellite performs an initial spiral thrusting manoeuvre to either increase or decrease its semi-major axis. It then drifts at this altitude for a given time to achieve the required separation, before performing a final spiral manoeuvre to reach the desired final altitude. The resultant change in RAAN or AoL is considered with respect to a non-manoeuvring reference satellite as illustrated in Fig. 1.

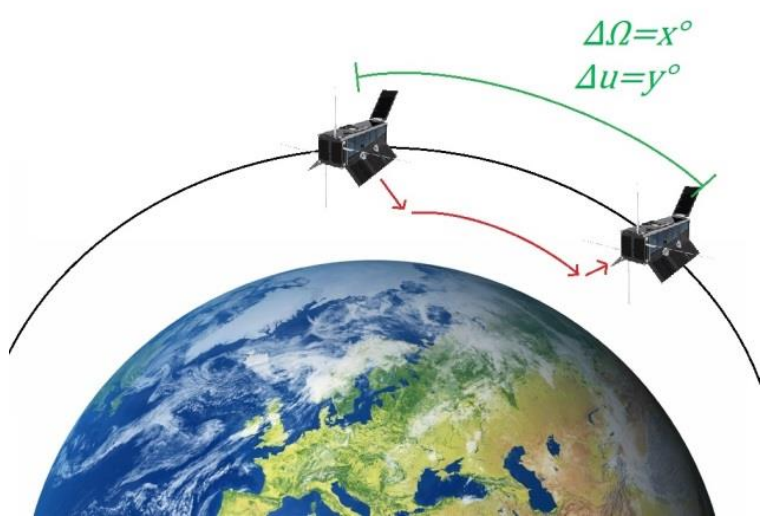

Fig. 1: Altitude lowering manoeuvre to separate through RAAN or AoL
While a change in either RAAN or AoL cannot in reality be performed independently of the other, the results previously presented by the authors show that, due to the relatively long manoeuvre time required to change a satellite's RAAN compared to the time required to change the AoL, the manoeuvres can be considered independently and the results later adapted to combine both [15].

The analytical method used is derived from the Gauss version of the Lagrange planetary equations [11] and considers a low-thrust manoeuvre with constant acceleration and no perturbations from drag or solar radiation pressure. It is assumed that the satellites maintain circular orbits throughout the entirety of the manoeuvre. These simplifications allow for the problem to be fully described and solved analytically.

The most general expression for the achievable change in RAAN, $\Delta \Omega$, is given by

$$
\begin{aligned}
\Delta \Omega=\frac{3}{256} \sqrt{\mu} \cos i J_{2} R_{e}^{2} & \left(\frac{\sqrt{\mu}}{16 A a_{0}^{4}}\left(\frac{\left(\mu+\beta a_{0}\right)^{4}}{\mu^{4}}-256\right)\right. \\
& -\frac{16 \sqrt{\mu}}{A}\left(\frac{1}{a_{3}^{4}}-\frac{\left(\beta+\frac{\mu}{a_{0}}\right)^{4}}{256 \mu^{4}}\right) \\
& +\left(\frac{\sqrt{\mu}}{A}\left(\frac{1}{\sqrt{a_{0}}}+\frac{1}{\sqrt{a_{3}}}-\frac{1}{\sqrt{\frac{\mu a_{0}}{\mu+\beta a_{0}}}}\right)\right. \\
& \left.\left.-t_{t}\right)\left(\frac{\mu a_{0}}{\mu+\beta a_{0}}\right)^{-7 / 2}+\frac{128 t_{t}}{a_{\mathrm{ref}}^{7 / 2}}\right)
\end{aligned}
$$

where

$$
\beta=\left(\sqrt{\frac{\mu}{a_{3}}} \pm \Delta \mathrm{V}_{t}\right)\left(2 \sqrt{\frac{\mu}{a_{0}}}+\sqrt{\frac{\mu}{a_{3}}} \pm \Delta \mathrm{V}_{t}\right)
$$

and $\mu$ is the standard gravitational parameter, $i$ is the inclination of the satellite's orbit, $J_{2}$ is the central body's second dynamic form factor, $R_{e}$ is the radius of the central body, $A$ is the acceleration produced by the propulsion system, $t_{t}$ is the total manoeuvre time, and $\Delta \mathrm{V}_{t}$ is the total change in velocity required for the satellite to complete the full manoeuvre. $a_{0}$ is the semi-major axis of the satellite at the beginning of the manoeuvre, $a_{3}$ is the desired final semi-major axis of the satellite, and $a_{r e f}$ is the semi-major axis of a nonmanoeuvring reference satellite against which the resultant change in RAAN is to be measured; in this case it is taken as $a_{3}$.

It should be noted that in equation 2, a ' + ' corresponds to the case where the satellite decreases its semi-major axis initially and increases its semi-major axis to reach its final orbit, and a '-. corresponds to the case where the satellite increases its semi-major axis initially and then decreases its 
semi-major axis to reach its final orbit. A positive $A$ value corresponds to an increase in semi-major axis, while a negative $A$ value corresponds to a reduction in semi-major axis.

The achievable change in AoL, $\Delta \mathrm{u}$, is given by

$$
\begin{aligned}
\Delta \mathrm{u}=\frac{1}{8}\left(\frac{8 \gamma \mu}{A a_{0}^{3 / 2}}-\frac{2 \gamma^{4} \mu}{A}\right. & -\frac{12 \gamma^{2} \mu}{A a_{0}}+\frac{8 \gamma^{3} \mu}{A \sqrt{a_{0}}} \\
& -\frac{2 \mu}{A}\left(\frac{\left(\mu+\beta a_{0}\right)^{2}}{16 \mu^{2} a_{0}^{2}}-\frac{1}{a_{3}^{2}}\right)-8 \sqrt{\frac{\mu}{a_{\mathrm{ref}}^{3}}} t_{t} \\
& +\sqrt{\frac{\left(\mu+\beta a_{0}\right)^{3}}{\mu^{2} a_{0}^{3}}}\left(t_{t}\right. \\
& \left.\left.-\frac{\sqrt{\mu}}{A}\left(\gamma+\frac{1}{\sqrt{a_{3}}}-\frac{1}{2 \sqrt{\frac{\mu a_{0}}{\mu+\beta a_{0}}}}\right)\right)\right)
\end{aligned}
$$

where, as before,

$$
\beta=\left(\sqrt{\frac{\mu}{a_{3}}} \pm \Delta \mathrm{V}_{t}\right)\left(2 \sqrt{\frac{\mu}{a_{0}}}+\sqrt{\frac{\mu}{a_{3}}} \pm \Delta \mathrm{V}_{t}\right)
$$

and

$$
\gamma=\left(\frac{1}{\sqrt{a_{0}}}-\frac{1}{2 \sqrt{\frac{\mu a_{0}}{\mu+\beta a_{0}}}}\right)
$$

with all symbols as previously defined, and the use of ' + ' and ' - ' as in the case of the RAAN separation manoeuvre.

In both cases, these general solutions can be reduced to represent specific simple manoeuvres by applying the relevant boundary conditions.

\section{MODEL VALIDATION}

In order to validate the model, the FORMOSAT-3/COSMIC constellation deployment was analysed using the analytical method described in Section II and the results compared with existing mission data. This constellation consists of six satellites which were initially launched into an approximately circular orbit with an altitude of $522 \mathrm{~km}$. The altitude of each satellite was then raised to $800 \mathrm{~km}$ with the manoeuvres timed to achieve a $-30^{\circ}$ RAAN separation between the satellites [9].

The six satellite manoeuvres were carried out over an 18 month period in 2006 and 2007. One of the satellites (FM3) experienced a solar array deployment failure and could not complete the orbit-raising manoeuvre. The other five satellites all reached the required final altitude and achieved the desired RAAN separation [10].

With knowledge of the initial and final semimajor axes of each satellite, and with the assumption of circular orbits and ignoring atmospheric drag, the required $\Delta \mathrm{V}$ can be calculated as $152.494 \mathrm{~m} / \mathrm{s}$ per satellite manoeuvre. This allows the achievable RAAN separation to be described as a function of the transfer time only, as shown in Fig. 2. Here, the total transfer time consists of the time spent in the initial orbit as well as the time required to complete the orbit-raising manoeuvre. The lines on the graph indicate the desired RAAN separations to be achieved and the corresponding total time as calculated using the analytical method.

Using the two-line element (TLE) data of the FORMOSAT-3/COSMIC satellites it is possible to track the satellites through their manoeuvres, as shown in Fig. 3, and thus to approximate the true time required to achieve the desired RAAN separation.

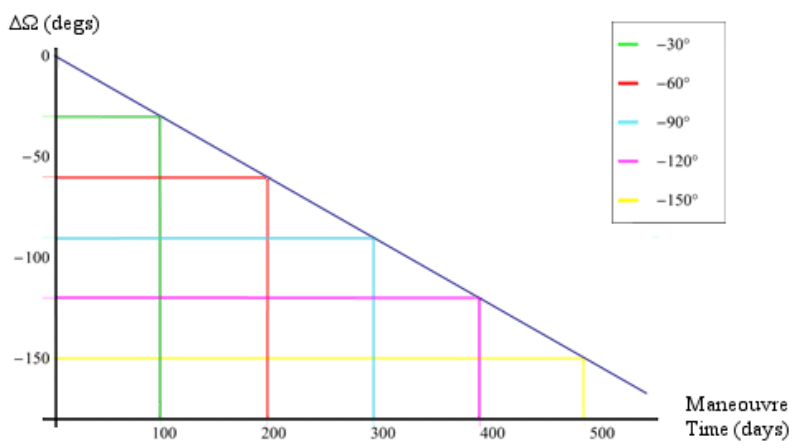

Fig. 2: Time required to achieve the desired separation between the FORMOSAT3/COSMIC constellation satellites

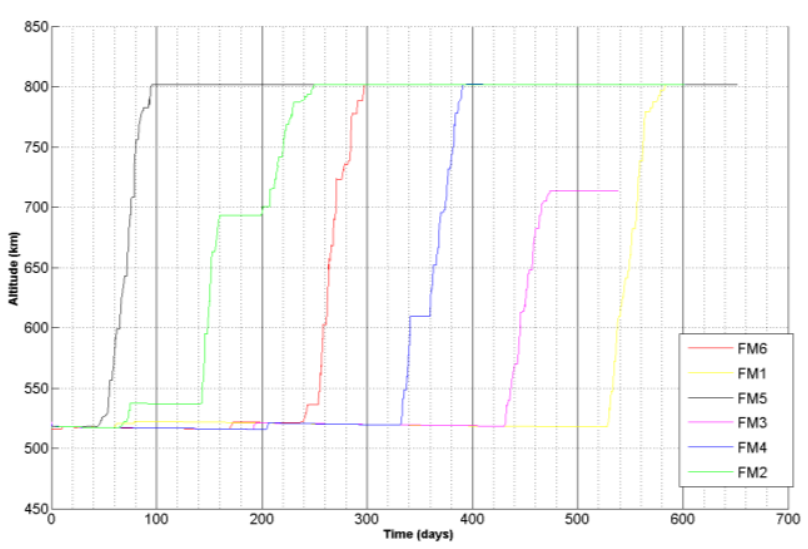

Fig. 3: TLE data from FORMOSAT-3/COSMIC constellation showing RAAN phasing manoeuvre 
To compare the calculated results with the actual results, the desired RAAN separation is defined in reference to the first satellite to be manoeuvred (FM5), which for the purposes of analysis is assumed to be the reference satellite. This means that the initial time, $t_{0}$, is taken as the time at which FM5 reaches its final orbit. This gives the time required to achieve the desired RAAN separations compared with the true manoeuvre time as shown in Table 1 . These results show that for FM6, FM4 and FM1 the proposed analytical method accurately predicts the time required to achieve the given RAAN separation with less than 5\% error. FM3 cannot be used for comparison as it never reached the desired final orbit altitude, and the error in the prediction of the time for FM2 can be explained by the approximately 40 day pause at $700 \mathrm{~km}$ altitude during the first manoeuvre. While the consideration of drag and other influences will likely give improved results, the current solution is considered to be sufficiently accurate to predict the required time and $\Delta \mathrm{V}$ for constellation deployment in the case of approximately circular orbits.

\begin{tabular}{llll}
\hline Spacecraft & $\begin{array}{l}\text { Desired RAAN } \\
\text { Separation w.r.t. } \\
\text { FM5 (degs) }\end{array}$ & $\begin{array}{l}\text { Calculated } \\
\text { total } \\
\text { manoeuvre } \\
\text { time (days) }\end{array}$ & $\begin{array}{l}\text { TLE Data } \\
\text { approximate } \\
\text { total manoeuvre } \\
\text { time (days) }\end{array}$ \\
\hline FM5 & 0 & 0 & 0 \\
FM2 & -30 & 97 & 150 \\
FM6 & -60 & 194 & 200 \\
FM4 & -90 & 291 & 290 \\
FM3 & -120 & 388 & - \\
FM1 & -150 & 484 & 480 \\
\hline
\end{tabular}

Table 1: Time required to achieve desired RAAN separation, calculated values versus true data

\section{CASE STUDY}

Having validated the analytical method, a case study was then carried out considering the deployment of a constellation of small satellites for earth observation.

Three different constellation designs are considered, the parameters for which are given in Table 2. The $\mathrm{t} / \mathrm{p} / \mathrm{f}$ value given corresponds to the Walker description of constellation design with $t$ being the total number of satellites in the constellation, $\mathrm{p}$ being the number of orbital planes and $\mathrm{f}$ denoting the phasing between satellites in neighbouring planes [16]. Walker Delta orbits are the general constellation geometry defined by these parameters and can sit at any inclination; Walker Star constellations are those in which all orbits are of polar, or near-polar, inclination. The proposed constellation designs contain a number of orbit planes in each category.

Designs 1 and 2 are the designs selected from the previous University of Strathclyde study as the best balance of utility to cost constellation designs, while Design 3 is another option which was explored as part of the study [8].

\begin{tabular}{lllll}
\hline $\begin{array}{l}\text { Design } \\
\text { No. }\end{array}$ & $\begin{array}{l}\text { Altitude } \\
(\mathrm{km})\end{array}$ & $\begin{array}{l}\text { Inclination } \\
(\mathrm{degs})\end{array}$ & $\begin{array}{l}\text { Delta } \\
\mathrm{t} / \mathrm{p} / \mathrm{f}\end{array}$ & $\begin{array}{l}\text { Star } \\
\mathrm{t} / \mathrm{p} / \mathrm{f}\end{array}$ \\
\hline 1 & 550 & 50 & $16 / 4 / 3$ & $6 / 2 / 1$ \\
2 & 550 & 60 & $20 / 4 / 2$ & $6 / 2 / 1$ \\
3 & 550 & 50 & $18 / 6 / 2$ & $6 / 2 / 1$ \\
\hline
\end{tabular}

Table 2: Constellation Design Parameters

\section{IV.I. Optimal Satellite Distribution}

In order to consider the deployment of a satellite constellation it is necessary to define the final positions of each of the individual satellites. Generally, satellite positions within a constellation are described relative to each other, as in the case of a Walker Delta or Walker Star constellation [1618]. However, it is also necessary to define the position of each satellite with respect to the launch injection point and, due to the lengthy manoeuvre times involved in changing the RAAN of the satellites, the positioning selected may have a large influence on the overall manoeuvre time and propellant cost. It has also been shown that for a given orbit, achieving a change in RAAN or AoL can be done more efficiently in one direction than in the other [15]. This means that evenly distributing the satellites from the launch injection point in both directions is unlikely to be the most efficient deployment method.

To find the ideal satellite distribution with regards to the launch injection point it is necessary to first define the spacing of the satellites relative to each other, again considering RAAN and AoL separately. If the satellites, or satellite planes, are evenly distributed this can be simply described by

$$
\Delta \Omega_{i}=\Delta \Omega_{1}+(i-1)\left(\frac{2 \pi}{n}\right)
$$

and

$$
\Delta \mathrm{u}_{i}=\Delta \mathrm{u}_{1}+(i-1)\left(\frac{2 \pi}{n}\right)
$$

for $i: 1 \rightarrow n$ where $i$ is the satellite number and $n$ is the total number of planes when considering RAAN distribution, or the number of satellites within a plane when considering AoL distribution. The two satellites positioned furthest from the manoeuvre starting point in this case will be satellite 1 and satellite $n$. By describing the change of RAAN or AoL of these two satellites analytically using equations 1 and 3 respectively, it is then possible to solve for the shortest time manoeuvre by setting the requirement that both satellites must reach their final position at the same time.

Note that the method described can be applied even if the satellites are not evenly distributed, but the position of the satellites relative to each other would need to be explicitly defined. 


\section{IV.II. Drag}

Accounting for atmospheric drag in an analytical solution is not straightforward as the effective drag force does not vary linearly with altitude. However, the general perturbations method provided by Kerr and Macdonald [19, 20] can be used with some simplification to determine if a satellite in the constellation will deorbit during deployment.

This method provides orbit lifetime predictions contingent on the launch date of a satellite as it includes an analytical atmospheric density model incorporating solar flux. As this study is a theoretical deployment strategy no launch date is known and therefore the solar flux is assumed to be constant at an average rate over the entire deployment time period. In reality some satellites in the constellation may deorbit more quickly than others depending on the solar flux conditions during the drift period, and this should be taken into consideration before applying this method to a proposed constellation design.

In order to account for atmospheric drag in the analytical deployment method presented, the satellite distribution and manoeuvre is considered excluding drag, and the drift phase is assumed to occur at a constant altitude. By making use of the analytical orbit lifetime prediction method it is then possible to calculate the true final altitude of each satellite at the end of the drift phase. Whilst this does not account for the variation in the rate of change of RAAN due to the change in altitude throughout the drift phase, it does ensure that none of the satellites deorbit during deployment and therefore that the constellation deployment strategy is feasible. In addition, the total $\Delta \mathrm{V}$ required for the manoeuvre is calculated using the post-drift altitude with drag taken into account.

\section{IV.III. Costing}

While there are CubeSat propulsion systems in development, it is currently unlikely that the necessary plane change manoeuvres described in Section II could be carried out by individual CubeSats due to the required $\Delta \mathrm{V}$ cost [6]. However, it would be possible to stow individual satellites on a larger carrier satellite which could deliver the satellites to the required orbit plane. From here, the satellites could be distributed within the plane using their own on-board propulsion or by using springs of varying strengths to control deployment [21].

Design, development and manufacture costs have already been considered as part of the previous University of Strathclyde study [8] and are assumed to be consistent regardless of whether traditional launch methods or the use of the proposed in-orbit deployment strategy is employed. As such, the costing done here focusses on the launch costs associated with both methods.
Two different launch providers are considered and the most applicable of their available launches selected to meet the mission requirements. These launch providers are Spaceflight Industries Inc. [22] and Firefly Space Systems [23]. Spaceflight Industries Inc. currently provide rideshare launch opportunities for small satellites; this means that the satellites would be considered secondary payloads and would have inexact knowledge of the final orbit and no control over the launch itself. Firefly Space Systems are in the process of developing a dedicated small satellite launch vehicle with a maximum payload of $400 \mathrm{~kg}$. This has the advantage of being able to provide dedicated launches, allowing the customer to choose their orbital parameters and launch schedule. However, as the cost in this case is per launch, rather than per satellite, the cost of the launch may be much higher than in the rideshare case unless the launch vehicle payload capacity is fully used or other satellites can be found to make use of the remaining payload capacity.

\section{IV.III.I Traditional Launch Methods}

Traditional launch methods here assume that no carrier vehicle is used and that the individual satellites have little to no manoeuvring capability. This means the satellites must be inserted at the correct altitude, inclination and RAAN by the launch vehicle. In this case, one launch will be required for each plane of the constellation. In the case of a rideshare launch the total launch cost is simply calculated as

$$
C_{\text {total }}=C_{\text {sat }} \times n \times p
$$

where $C_{s a t}$ is the launch cost per satellite, $n$ is the number of satellites in each plane and $p$ is the total number of orbit planes. In the case of a dedicated launch the cost would be

$$
C_{\text {total }}=C_{\text {launch }} \times p
$$

where $C_{\text {launch }}$ is the cost of a single dedicated launch.

\section{IV.III.II Carrier Vehicle Method}

In the case of manoeuvrable carrier vehicles being used to deploy the constellation, the number of launches required to place all satellites into orbit will be dependent on the number of satellites to be launched and the maximum payload capabilities of the launch vehicle. In the case of a rideshare launch the total launch cost will be calculated as

$$
C_{\text {total }}=C_{\text {carrier }} \times p
$$

where $C_{\text {carrier }}$ is the launch cost per carrier vehicle. In the case of a dedicated launch the cost would be 


$$
C_{\text {total }}=C_{\text {launch }}
$$

where $C_{\text {launch }}$ is the cost of a single dedicated launch.

The size of the carrier vehicle will be primarily dependent on the manoeuvre it is required to perform and the number of spacecraft it is required carry. As an initial estimate the dry mass of the carrier is estimated as

$$
m_{f}=m_{p} \times 3.3
$$

where $m_{p}$ is the mass of the satellites to be carried [24]. A low power Xenon resistojet propulsion system is considered as a baseline with a specific impulse of $48 \mathrm{secs}$ and the ability to deliver up to $100 \mathrm{mN}$ thrust [25].

From this, the maximum allowable propellant mass is calculated to make use of the full payload mass available on the dedicated launch vehicle, and the maximum allowable $\Delta \mathrm{V}$ calculated from this using the rocket equation [26]. A margin of $20 \%$ is applied to both the spacecraft total mass and the $\Delta \mathrm{V}$ calculation to ensure a conservative estimate.

It is assumed for these analyses that one carrier vehicle is used per orbital plane; while it would be possible to use one carrier to deliver satellites to multiple orbit planes, the length of time required to deploy the constellation using a single carrier vehicle would in all cases be longer than when using one carrier per plane and as such it is not considered in this study.

\section{RESULTS}

\section{$\underline{\text { V.I. Optimal Satellite Distribution }}$}

\section{V.I.I RAAN Separation}

In the case used for analysis, four orbital planes are considered which are evenly distributed through $360^{\circ}$ (i.e. $90^{\circ}$ separation between each plane). The mission parameters are given in Table 3 and Table 4.

It is assumed that of the furthest two satellites to be placed, satellite 1 and satellite 4 , satellite 1 will initially lower its semi-major axis, resulting in a negative $\Delta \Omega$, and satellite 4 will initially raise its semi-major axis above the final desired altitude resulting in a positive $\Delta \Omega$.

As the required separation of satellite 1 and satellite 4 is known to be $270^{\circ}$, by plotting the achievable $\Delta \Omega$ of satellite 1 against the achievable $\Delta \Omega$ of satellite 4 minus the required $270^{\circ}$ separation as shown in Fig. 4, an intersection can be found along which both satellites will arrive at their required final position at the same time. The time at which this occurs is dependent on the total $\Delta \mathrm{V}$ used for the manoeuvre.
Once a position for these first two satellites has been selected, the position of the other satellites will be decided relative to them. The time required to place the remaining satellites in position will be dependent on the $\Delta \mathrm{V}$ selected, but in any case will be shorter than the time required for the first two satellites to reach their final positions.

\section{V.I.II Argument of Latitude Separation}

In considering the placement of the satellites with regard to argument of latitude, four satellites are considered for even distribution within each orbital plane, corresponding to $90^{\circ}$ separation between each satellite. For an initial analysis it is assumed that of the furthest two satellites to be placed, satellite 1 and satellite 4 , satellite 1 will initially lower its semi-major axis, resulting in a positive $\Delta \mathrm{u}$, and satellite 4 will initially raise its semi-major axis above the final desired altitude resulting in a negative $\Delta \mathrm{u}$.

\begin{tabular}{lcll}
\hline Parameter & Symbol & Value & Units \\
\hline $\begin{array}{l}\text { Gravitational } \\
\text { Parameter }\end{array}$ & $\mu$ & $3.986 \mathrm{E} 14$ & $\mathrm{~m}^{3} / \mathrm{s}^{2}$ \\
Radius of Earth & $\mathrm{R}_{\mathrm{e}}$ & $6.371 \mathrm{E} 3$ & $\mathrm{~km}$ \\
$\mathrm{~J}_{2}$ Parameter & $\mathrm{J}_{2}$ & $1.0827 \mathrm{E}-3$ & - \\
\hline
\end{tabular}

Table 3: Orbital Constants

\begin{tabular}{lccc}
\hline Parameter & Symbol & Value & Units \\
\hline $\begin{array}{l}\text { Propulsion } \\
\text { acceleration }\end{array}$ & $\mathrm{A}$ & \pm 0.001 & $\mathrm{~m} / \mathrm{s}^{2}$ \\
$\begin{array}{l}\text { Inclination } \\
\begin{array}{l}\text { Initial semi- } \\
\text { major axis }\end{array}\end{array}$ & $a_{0}$ & 6771 & $\mathrm{~km}$ \\
$\begin{array}{l}\text { Final semi- } \\
\text { major axis }\end{array}$ & $a_{3}$ & 6921 & $\mathrm{~km}$ \\
\hline
\end{tabular}

Table 4: RAAN Analysis Mission Parameters

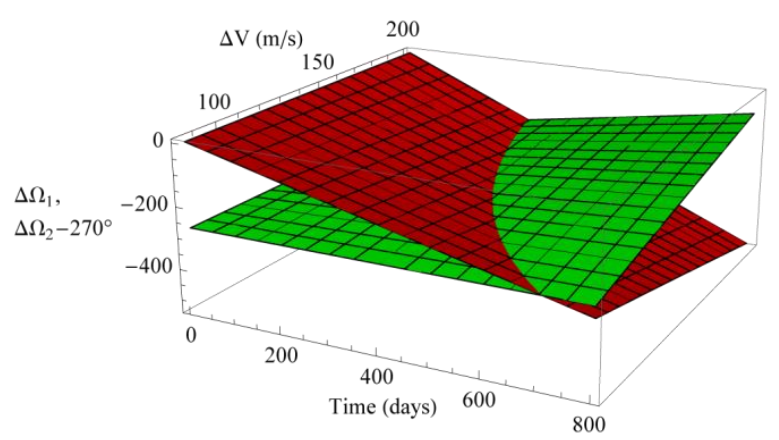

Fig. 4: Optimal $\Delta \Omega$ of satellite 1 (red) and the relative $\Delta \Omega$ of satellite 4 (green) as a function of $\Delta \mathrm{V}$ and manoeuvre time 
For this case it is assumed that the satellites begin their manoeuvres at the desired final altitude. This is in accordance with the recommendation made in the authors' previous work that the RAAN distribution manoeuvre be completed first and then the AoL manoeuvre carried out [15]. The orbital constants are as in the case of the RAAN separation and are given in in Table 3 and the mission parameters are as given in Table 5 .

Similar to the case of the RAAN distribution the achievable $\Delta \mathrm{u}$ of satellite 1 is plotted against the achievable $\Delta \mathrm{u}$ of satellite 4 minus $270^{\circ}$ as shown in Fig. 5. Here the results are only plotted for cases in which the total manoeuvre time is greater than the necessary thrust time in order to show only realistic scenarios.

In this case the graphs do not intersect indicating that when distributing the satellites within the plane for this constellation, it will always be more efficient to lower the altitude of the satellite initially and move all satellites in the same direction through a positve $\Delta \mathrm{u}$. Thus the minimum time manoeuvre would correspond to a case in which one satellite remains at the initial location and the other satellites are moved relative to it.

\begin{tabular}{lccc}
\hline Parameter & Symbol & Value & Units \\
\hline $\begin{array}{l}\text { Propulsion } \\
\text { acceleration }\end{array}$ & $\mathrm{A}$ & \pm 0.001 & $\mathrm{~m} / \mathrm{s}^{2}$ \\
$\begin{array}{l}\text { Inclination } \\
\begin{array}{l}\text { Initial semi- } \\
\text { major axis }\end{array}\end{array}$ & $a_{0}$ & 6921 & $\mathrm{~km}$ \\
$\begin{array}{l}\text { Final semi- } \\
\text { major axis }\end{array}$ & $a_{3}$ & 6921 & $\mathrm{~km}$ \\
\hline
\end{tabular}

Table 5: AoL Analysis Mission Parameters

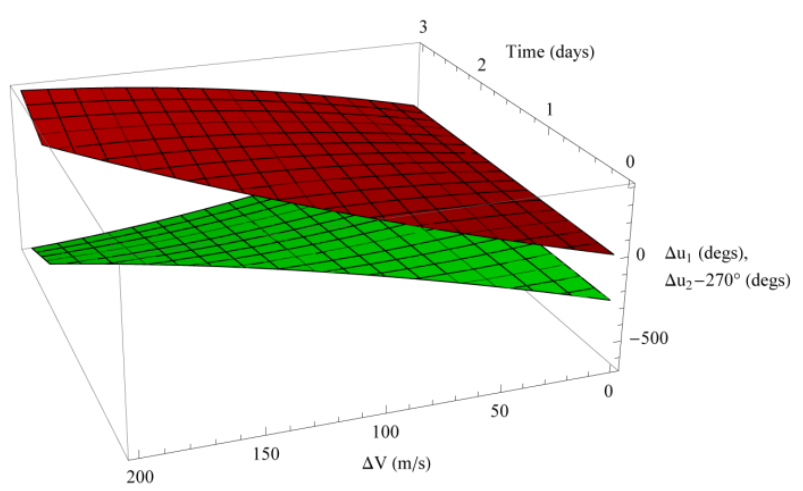

Fig. 5: Optimal $\Delta \mathrm{u}$ of satellite 1 (red) and the relative $\Delta u$ of satellite 4 (green) as a function of $\Delta \mathrm{V}$ and manoeuvre time

\section{V.II. Drag}

During the proposed satellite manoeuvres, the satellites will spend a relatively long time in the drift orbit before manoeuvring to reach the desired final altitude. For satellites lowering their altitude in this phase the effects of atmospheric drag must be considered to ensure they do not deorbit during this drift period. In addition, the $\Delta \mathrm{V}$ required to reach the desired constellation altitude will be dependent on the altitude of the satellite at the end of the drift phase.

The results of a general analysis are shown in Fig. 6. From this it is clear that the lower the drift orbit and the longer the satellite spends in this drift phase, the greater the influence of atmospheric drag. As a result of this analysis, combined with the deployment scenario results from Section V.I.I, it is decided that for the case study considered the satellites should be launched to an initial altitude of $550 \mathrm{~km}$ to prevent them from deorbiting before the full constellation can be deployed.

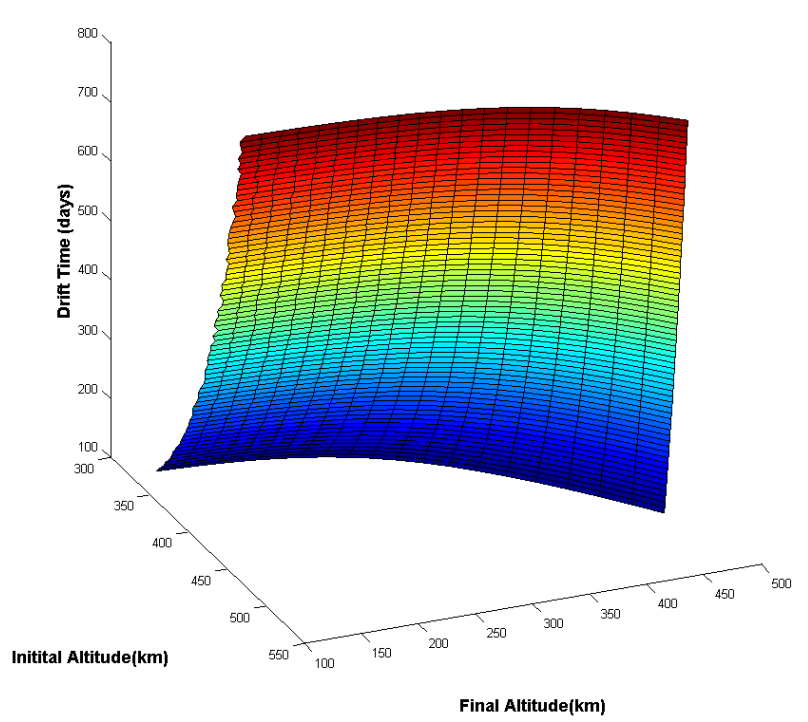

Fig. 6: Final altitude as a function of initial altitude and dift time

\section{V.III. Costing}

The launch costs of both launch service providers considered are given in Table 6 and Table 7, as well the most appropriate orbital parameters that can be provided by the launch vehicle for the three constellation designs considered. In the case of Spaceflight Industries Inc. the cost per kilogram is calculated for satellite launches carrying the closest mass to that of all carriers to be launched. The payload capacity of the Firefly Space Systems dedicated launch vehicle is dependent on the altitude and inclination of the launch injection orbit [27]. 


\begin{tabular}{llllll}
\hline $\begin{array}{l}\text { Spaceflight } \\
\text { Industries Inc. }\end{array}$ & $\begin{array}{l}\text { Launch } \\
\text { Type }\end{array}$ & $\begin{array}{l}\text { Cost per 6U } \\
\text { CubeSat }(£)\end{array}$ & $\begin{array}{l}\text { Cost per kg } \\
\text { Delta/Star }(£)\end{array}$ & $\begin{array}{l}\text { Launch Altitude } \\
\text { Delta/Star }(\mathrm{km})\end{array}$ & $\begin{array}{l}\text { Launch Inclination } \\
\text { Delta/Star }(\mathrm{degs})\end{array}$ \\
\hline Design 1 \& 3 & Rideshare & 354,250 & $19,067 / 25,277$ & $400 / 510$ & $51.6 / \mathrm{SSO}(\approx 97.41)$ \\
Design 2 & Rideshare & 354,250 & $18,200 / 25,277$ & $500-600 / 600$ & $63.4 / \mathrm{SSO}(\approx 97.76)$ \\
\hline
\end{tabular}

Table 6: Spaceflight Industries Inc. Launch Vehicle Datasheet

\begin{tabular}{llllll}
\hline Firefly Space & Launch & Cost per & Launch Altitude & Launch Inclination & Max Payload Mass \\
Systems & Type & launch $(£)$ & Delta/ Star $(\mathrm{km})$ & Delta $($ degs $)$ & Delta / Star $(\mathrm{kg})$ \\
\hline Design 1 \& 3 & Dedicated & $5,200,000$ & $550 / 550$ & $50 / 90$ & $315 / 215$ \\
Design 2 & Dedicated & $5,200,000$ & $550 / 550$ & $60 / 90$ & $280 / 215$ \\
\hline
\end{tabular}

Table 7: Firefly Space Systems Launch Vehicle Datasheet

\section{V.III.I Traditional Launch Methods}

If using the Spaceflight Industries Inc. rideshare launches, the total cost for launching the entire constellation is calculated to be $£ 7.79$ million for Design 1, £9.21million for Design 2 and $£ 8.5$ million for Design 3 when using traditional launch methods.

Using the dedicated launch vehicle provided by Firefly Space Services the launch cost is calculated to be $£ 31.2$ million in the case of Design 1 and Design 2, assuming that the remaining payload space is not filled by another satellite. The Design 3 launch cost is calculated as $£ 41$.2million.

\section{V.III.II Carrier Vehicle Method}

The calculated carrier vehicle parameters are given in Table 8 for the case in which Firefly Space Systems dedicated launch vehicle is used. The same carrier vehicle mass is assumed for the rideshare launch.

From the maximum allowable propellant mass it is possible to estimate the maximum allowable $\Delta \mathrm{V}$ for each carrier. From this, the optimal distribution of the constellation orbital planes, as well as the total time required to deploy each satellite can be calculated. These results are shown in Table 9 for the Walker Delta Orbits considered. The actual manoeuvre $\Delta \mathrm{V}$ value listed in Table 9 differs from the allowable value shown in Table 8 in some cases. This is because the actual value is the maximum value which can be used without the satellite deorbiting during the deployment manoeuvre as a result of atmospheric drag due to the low altitude of the drift orbit. In the case of the Walker Star orbits, which are to be placed at $90^{\circ}$ inclination, the time required to separate the orbital planes by the required amount is such that the satellites would deorbit before the manoeuvre could be completed. Thus it is assumed that one launch would be required to populate each Walker Star orbit plane individually.

These results show that while each carrier in all three constellation designs uses a similar $\Delta \mathrm{V}$ value, the time to deployment varies greatly. In the case of Design 2 this is because the RAAN change manoeuvre is naturally slower at the higher inclination [11]. In Design 3, the greater number of orbital planes means the satellites must travel further to reach their final position.

Using the Spaceflight Industries Inc. rideshare launches, the total cost for launching the carrier vehicles is calculated to be $£ 22.9$ million for Design 1 and 3 , and $£ 31.2$ million for Design 2 .

The cost of deployment using the Firefly Space Systems dedicated launch is calculated as $£ 20$.8million for Design 1 and Design 3 and $£ 31.2$ million for Design 2 .

The costs of all methods considered are summarised in Table 10 from which it can be seen that traditional launch methods utilising rideshare opportunities offer the most economical means of constellation deployment. However this has the disadvantage of allowing the customer minimal control over the orbit parameters and launch schedule. In addition it can be seen in Table 6 that to achieve an orbit inclination of $50^{\circ}$ using the rideshare launches requires that the satellites be launched to just $400 \mathrm{~km}$ altitude. As previously shown in Section V.II this would result in a very short orbit lifetime due to the effects of atmospheric drag. When considering a dedicated launch, deployment using carrier vehicles offers a significant cost reduction when compared with traditional methods.

\begin{tabular}{lcccc}
\hline $\begin{array}{l}\text { Firefly Space } \\
\text { Systems }\end{array}$ & $\begin{array}{l}\text { Carriers per } \\
\text { Launch }\end{array}$ & $\begin{array}{l}\text { Carrier dry mass } \\
(\mathrm{kg})\end{array}$ & $\begin{array}{l}\text { Carrier allowable } \\
\text { propellant mass }(\mathrm{kg})\end{array}$ & $\begin{array}{l}\text { Carrier allowable } \Delta \mathrm{V} \\
(\mathrm{m} / \mathrm{s})\end{array}$ \\
\hline Design 1 & 2 & 79.2 & 52.05 & 198 \\
Design 2 & 1 & 99 & 134.3 & 336 \\
Design 3 & 3 & 59.4 & 28.1 & 152 \\
Star Orbits 1, 2 \& 3 & 1 & 118.8 & 60.4 & 161 \\
\hline
\end{tabular}

Table 8: Carrier Vehicle Parameters for Various Constellation Designs 


\begin{tabular}{|c|c|c|c|c|c|c|c|c|c|c|c|c|c|}
\hline \multirow{2}{*}{ Design 1} & \multicolumn{6}{|c|}{ RAAN of Orbital Planes (degs) } & \multicolumn{6}{|c|}{ Manoeuvre time for carrier (days) } & Actual $\Delta \mathrm{V}(\mathrm{m} / \mathrm{s})$ \\
\hline & -139 & & -49 & 41 & & 131 & 439 & & 155 & 136 & & 439 & 187 \\
\hline Design 2 & -138 & & -48 & 42 & & 132 & 740 & & 257 & 236 & & 740 & 160 \\
\hline Design 3 & -153 & -93 & -33 & 26 & 86 & 146 & 616 & 375 & 134 & 113 & 365 & 616 & 152 \\
\hline
\end{tabular}

Table 9: Deployment Results for Various Constellation Designs

\begin{tabular}{|c|c|c|c|c|}
\hline \multirow{2}{*}{ Cost (million $£)$} & \multicolumn{2}{|c|}{ Traditional Launch Method } & \multicolumn{2}{|c|}{ Carrier Vehicle Method } \\
\hline & Rideshare & Dedicated & Rideshare & Dedicated \\
\hline Design 1 & 7.79 & 31.2 & 22.9 & 20.8 \\
\hline Design 2 & 9.21 & 31.2 & 31.2 & 31.2 \\
\hline Design 3 & 8.5 & 41.2 & 22.9 & 20.8 \\
\hline
\end{tabular}

Table 10: Cost for various launch methods and constellation designs

\section{CONCLUSION}

Using carrier vehicles to deploy a constellation of CubeSats is shown to be a practical alternative to traditional launch methods. Optimising the position of each satellite to be deployed allows for the total deployment time to be minimised and considering the effect of atmospheric drag ensures that the manoeuvres and associated propellant costs are realistic. While the time for deployment can be lengthy depending on the $\Delta \mathrm{V}$ used, planning for this could allow a limited service to start once some satellites are in place. This deployment strategy would also enhance system responsiveness by allowing for the deployment to be adapted in the face of changing mission requirements and removing the reliance of the mission on uncertain launch schedules.

The case study considered demonstrates that a constellation of CubeSats for earth observation could be deployed by carrier vehicles using existing propulsion systems and launch vehicles. While the cost of launching individual CubeSats using rideshare launches is identified as the lowest cost scenario, it reduces the usefulness of the constellation significantly due to the lack of control over each satellite's final position as well as the launch schedule. Launching carrier vehicles on rideshare launches would partially combat this by allowing the satellites to manoeuvre after launch, but the increase in cost is significant.

Launching the carrier vehicles using a dedicated launcher costs less than launching them via rideshare, and also costs significantly less than launching the individual satellites using a dedicated launch vehicle. This would give the customer full control over their launch injection and schedule and so is recommended for deploying a constellation of this kind.

The greatest reduction in cost when using the carrier vehicles comes when considering constellations with a large number of planes, as shown by the significant reduction in cost when comparing the launch of Design 3 using carrier vehicles versus traditional launch methods. The use of the carriers could allow for constellation designs with a large number of planes to be implemented at little or no increase in cost compared to those with fewer planes, and as such may allow for increased system performance and greater mission flexibility.

\section{REFERENCES}

[1] Radius Space (2015). Nanosatellite Database. Available: http://www.nanosats.eu/

[2] J. Tristancho Martínez, "Implementation of a femto-satellite and a mini-launcher for the N Prize," 2010.

[3] J. Bouwmeester and J. Guo, "Survey of worldwide pico-and nanosatellite missions, distributions and subsystem technology," Acta Astronautica, vol. 67, pp. 854-862, 2010.

[4] D. Selva and D. Krejci, "A survey and assessment of the capabilities of Cubesats for Earth observation," Acta Astronautica, vol. 74, pp. 50-68, 2012.

[5] European Space Agency. Announcement of Opportunity \#2 'Nanosatellite constellations for Earth Observation'. Available:

http://gsp.esa.int/web/guest/ao2_descriptio $\mathrm{n}$

[6] K. Woellert, P. Ehrenfreund, A. J. Ricco, and H. Hertzfeld, "Cubesats: Costeffective science and technology platforms for emerging and developing nations," Advances in Space Research, vol. 47, pp. 663-684, 2011.

[7] S. Müncheberg, M. Krischke, and N. Lemke, "Nanosatellites and micro systems 
technology - capabilities, limitations and applications," Acta Astronautica, vol. 39, pp. 799-808, 1996.

[8] C. Lowe, M. Macdonald, C. Rogers, R. Thompson, M. Jessup, S. Wakelam, et al., "SysN.TN.004 Mission Design Report," University of Strathclyde, Scisys, Chemring Technolgy Solutions 2015.

[9] C.-J. Fong, C.-Y. Huang, V. Chu, N. Yen, Y.-H. Kuo, Y.-A. Liou, et al., "Mission results from FORMOSAT-3/COSMIC constellation system," Journal of Spacecraft and Rockets, vol. 45, pp. 12931302, 2008.

[10] C.-J. Fong, W.-T. Shiau, C.-T. Lin, T.-C. Kuo, C.-H. Chu, S.-K. Yang, et al., "Constellation deployment for the FORMOSAT-3/COSMIC mission," Geoscience and Remote Sensing, IEEE Transactions on, vol. 46, pp. 3367-3379, 2008.

[11] D. A. Vallado and W. D. McClain, Fundamentals of astrodynamics and applications vol. 12: Springer Science \& Business Media, 2001.

[12] O. L. De Weck, U. Scialom, and A. Siddiqi, "Optimal reconfiguration of satellite constellations with the auction algorithm," Acta Astronautica, vol. 62, pp. 112-130, 2008.

[13] L. Appel, M. Guelman, and D. Mishne, "Optimization of satellite constellation reconfiguration maneuvers," Acta Astronautica, vol. 99, pp. 166-174, 2014.

[14] N. Crisp, K. Smith, and P. Hollingsworth, "Launch and deployment of distributed small satellite systems," Acta Astronautica, vol. 114, pp. 65-78, 2015.

[15] C. McGrath and M. Macdonald, "Design of a Reconfigurable Satellite Constellation," presented at the 66th International Astronautical Congress, Jerusalem, Israel, 2015.

[16] P. Fortescue, G. Swinerd, and J. Stark, Spacecraft systems engineering, 3rd ed.: John Wiley \& Sons, 2011.

[17] J. G. Walker, "Circular orbit patterns providing continuous whole earth coverage," DTIC Document1970.

[18] J. G. Walker, "Continuous whole-earth coverage by circular-orbit satellite patterns," DTIC Document1977.

[19] E. Kerr and M. Macdonald, "Incorporating Solar Activity into General Perturbations Analysis of Artificial Satellite's Orbit Lifetime," In Draft for: Proceedings of the Royal Society A, 2015.

[20] E. Kerr and M. Macdonald, "A general perturbations method for spacecraft lifetime analysis," in 25th AAS/AIAA Space Flight Mechanics Meeting, 2015.

[21] J. A. King and N. J. Beidleman, "Method and apparatus for deploying a satellite network," ed: Google Patents, 1993.

[22] Spaceflight Industries Inc. (2015). Available: http://www.spaceflight.com/

[23] Firefly Space Systems (2015). Available: http://www.fireflyspace.com/

[24] W. J. Larson and J. R. Wertz, "Space mission analysis and design," Microcosm, Inc., Torrance, CA (US)1992.

[25] "Low Power Resistoject," vol. 0072017 v005, S. S. T. Ltd, Ed., ed, 2014.

[26] M. Macdonald and V. Badescu, The International Handbook of Space Technology: Springer, 2014.

[27] Firefly Alpha Payload User Guide (2015). Available: http://www.fireflyspace.com/downloads 